\title{
Light on the Biomineralization of Ferritin
}

Surya Narayanan ${ }^{1}$, Emre Firlar ${ }^{1,2}$, Tara Foroozan ${ }^{2}$, Nasim Farajpour ${ }^{3}$, Leigha Covnot $^{1}$, Reza Shahbazian Yassar $^{2}$ and Tolou Shokuhfar ${ }^{1,4}$

1. University of Illinois at Chicago, Department of Bioengineering, Chicago, IL.

2. University of Illinois at Chicago, Department of Industrial and Mechanical Engineering, Chicago, IL.

3. University of Illinois at Chicago, Department of Electrical and Computer Engineering, Chicago, IL.

4. University of Illinois at Chicago, College of Dentistry, Chicago, IL.

Nature and biology has always inspired scientists to understand and mimic the biological processes in $e x$ situ conditions. Biomineralization is one such aspect that is widely studied and focuses on understanding the formation of mineral in a controlled biological environment. There are different spectroscopy and transmission electron microscopy (TEM) based techniques which shines light on the biomineralization aspects, however there are still unanswered questions. Iron oxides formation is one such example and is observed in different life forms from lowest to highest order of magnitude including human beings.

External factors such as $\mathrm{pH}$, temperature, presence of organic salts influence the formation of different iron oxides. These factors impact human health and are important to be understood to explain the causes of many diseases and cures to treat them. For instance, ferritin is an iron storage protein present in many organisms including human beings. Despite the iron regulation mechanism in ferritin, iron accumulation and toxicity is witnessed in the diseased sites of the organ. Furthermore, iron oxides that are formed shows differences in crystallinity and structure [1].

Figure 1 shows the proposed regulation mechanism in ferritin. The conversion of $\mathrm{Fe}^{+2}$ ions to $\mathrm{Fe}_{2} \mathrm{O}_{3}(\mathrm{H} 2 \mathrm{O})$ $x$ happens through series of reactions [2]. Further, nucleation of the iron oxide clusters results in the ferritin core formation. Although the general chemistry of iron core formation is known, there are many unanswered questions such as the type of iron oxides formed as a consequence of the rate of biomineralization, and the microenvironment that exists within the protein structure.

With the conventional TEM, it was possible to observe the changes in the morphology of the ferritin structure with respect to the number of iron atoms that form the core structure [3]. However, sample preparation involves fixation and staining. To overcome these steps causing changes in the structure and to maintain the proteins in liquid environment, graphene liquid cells (GLCs) were introduced. GLCs offer advantages including low substrate thickness and being electron transparent [4]. Figure 2 shows the high angle annular dark field - scanning TEM (HAADF-STEM) image of human spleen ferritins in GLCs prepared as described by Wang et al, 2014 [5].

Through the high resolution STEM (HR-STEM) images obtained from different morphologies of ferritin it was observed that the crystal structure is polycrystalline with many irregularities. Further, the interplanar spacing of $0.14 \mathrm{~nm}, 0.24 \mathrm{~nm}, 0.26 \mathrm{~nm}$ and $0.27 \mathrm{~nm}$ in different ferritin structures indicated the presence of hematite, maghemite, magnetite, ferrihydrite, and goethite based on the differences in the the morphologies of ferritin. The types of crystals formed reflects the stages of the biomineralization. While hematite and magnetite are stable crystals, ferrihydrite is represented as a labile form of crystal. Likewise, goethite is the intermediate iron oxide formed which is later converted to hematite. This was also observed in the selected area electron diffraction (SAED) collected from the particles which indicated the rate of 
biomineralization. As a long term goal, benchtop chemistry based techniques will be applied in TEM scale to study the biomineralization in ferritin [6].

\section{References:}

[1] Collingwood J.F. et al, Journal of Alzheimer's Disease 14 (2008), p. 235.

[2] Bernacchioni C. et al, RSC Advances 6 (2016), p. 21219.

[3] Ying-His-Pan et al, Journal of Structural Biology 166 (2009), p. 22.

[4] Cho Hoduk, et al, Nano Lett 17 (2017), p. 414.

[5] Wang C. et al, Advanced Materials 26 (2014), p. 3410

[6] The authors acknowledge funding from the National Science Foundation - CAREER award- Grant No- DMR 1564950. Prof. Tolou Shokuhfar is acknowledged for her many useful discussions and contribution to this work. This work made use of instruments in the Electron Microscopy Service (Research Resources Center, UIC).

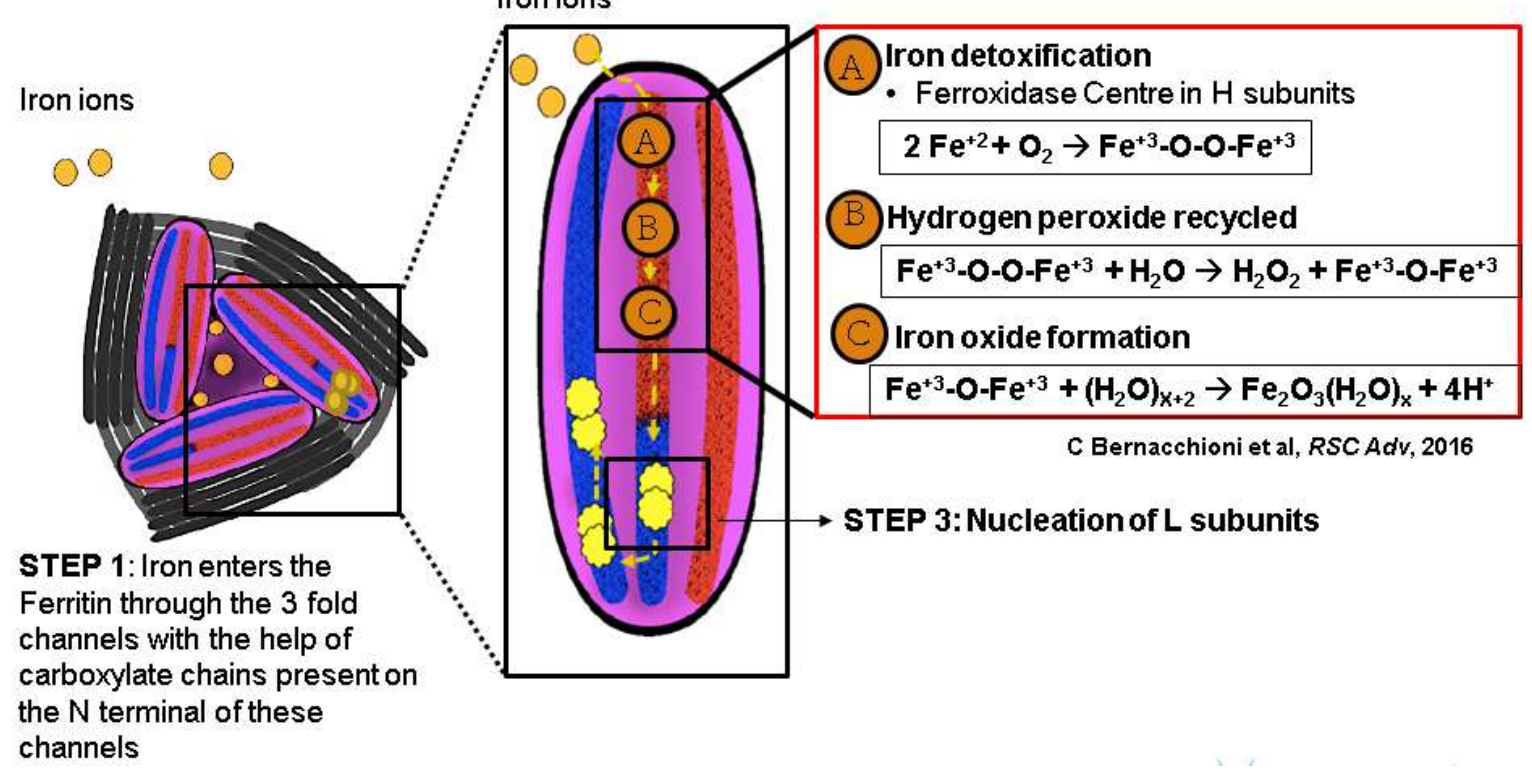

Figure 1. Shows the proposed iron regulation mechanism of Ferritin

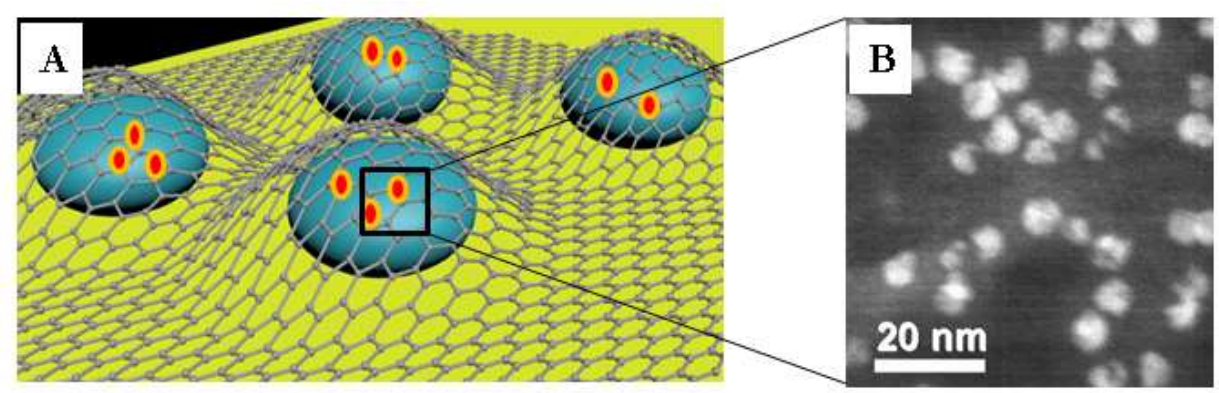

Figure 2. A) Schematic showing encapsulation of ferritins in GLCs B) HAADF STEM image of ferritin (Scale: $20 \mathrm{~nm}$ ) 